\title{
The Impact of Overlap Regions in Cognitive Radio Networks
}

\author{
Minh Thao QUACH \\ LaBRI - Université Bordeaux 1, Bordeaux, France \\ Email: minh-thao.quach@labri.fr
}

\author{
Hicham KHALIFE \\ Thales Communications \& Security, Colombes, France \\ Email: hicham.khalife@thalesgroup.com
}

\begin{abstract}
In this paper, we evaluate the impact of cognitive radio transmissions on primary radio communications. Our objective is to characterize precisely, based on the position of secondary users, the vulnerability areas where interference to primary radios can be generated. To do so, we detail all the cases where overlap regions between primary and secondary transmitters exist and derive the vulnerability area for each case. Through OMNeT++ simulations, we illustrate that there is a dependency between the size of the overlap area and the impact on the primary receivers: the smaller the overlap region, the smaller the impact on the receivers in this area. Furthermore, an efficient channel selection by cognitive radios can reduce by $40 \%$ the interference observed on primary receivers.
\end{abstract}

\section{INTRODUCTION}

We characterize the impact of secondary transmitters on primary radios in cognitive radio networks when a coverage overlap between the cognitive radio $(\mathrm{CR})$ and primary radio (PR) transmitters exists. In practice, accurately measuring this overlap and its effect on every available channel is necessary for effective spectrum selection. All possible overlap scenarios between PR and CR transmitters are identified, and the vulnerability region is mathematically computed. Our technique requires only the co-ordinate position and coverage area (transmission power) of CRs and PRs in order to compute the region where the two zones geographically overlap.

The size of the obtained area is directly linked to the interference on the existing PR receivers, meaning that the smallest overlapping area causes the least interference to ongoing PR communications. Practically, if no PR receivers exist nor operate within the intersection area, the radio resources can be transparently used by the CR. Nevertheless, a clever channel selection which creates minimum overlap with the PR transmitter can guarantee that the interference to the PR receivers is minimal. This overlap information is considered as a valuable input metric for numerous mechanisms and protocols developed for cognitive radio networks such as spectrum management and routing solutions.

An overlapping perspective in Cognitive Radio Networks was proposed in [1], where the time overlap during sensing between the CRs was derived. The solution aimed at exploiting the waiting time for sensing while the other CRs report to a fusion center. Another work on the channel overlapping [2] discussed the partial frequency barrier overlapping when $\mathrm{PR}$ and $\mathrm{CR}$ coexist over the same frequency. PR receiver protection was studied in [3] in which a routing solution with considerations about overlapping was proposed. The routing mechanism ensures a perfect protection for PRs by selecting routes that avoid any overlap between primary radios and secondary radios coverage. Consequently, The resources of the overlap region may be usable when the $\mathrm{PR}$ receivers are inactive or inexistent. The broader aspect has not been investigated, so in this paper, we concentrate on studying the potential impact on the receiver devices within the overlap region. Relying on the results we provide, the spectrum usage can be optimized in this overlap area of congnitive radio networks.

\section{OVERLAP REGION IN COGNITIVE RADIO NETWORKS}

We call an overlap region the intersection of the two disks created by the coverage area of a cognitive radio transmitter and primary radio transmitter. The circumstances where the overlaps happen the impact on the primary receivers are then investigated. Additionally, by reducing this vulnerability area (i.e. the intersection between the communication ranges), $\mathrm{CR}$ performance can also be enhanced. In a cognitive radio environment, our computation targets the interference to the $\mathrm{PR}$ systems and then returns to the CR emitter the frequency where the overlap region is minimal.

In this study, we consider a single primary transmitter and a single cognitive node. We also assume the coverage area of $\mathrm{PR}$ and CR transmitters forms a perfect circle whose center is located at the position of the emitter. We denote by $O_{P}$ of coordinates $\left(x_{P}, y_{P}\right)$ and $O_{C}$ with $\left(x_{C}, y_{C}\right)$ the position of the PR and CR transmitters respectively, $d$ the distance between these two points and $R_{P} R_{C}$ the radius of their coverage disk respectively.

The location of the PR transmitter is assumed to be at the center of the $2 \mathrm{D}$ co-ordinate system (i.e. $x_{P}=y_{P}=0$ ), the Euclidean distance between PR and CR can be easily obtained $\left(d=\sqrt{x_{C}^{2}+y_{C}^{2}}\right)$. Note here that this assumption is only made in order to obtain compact equations and can be easily relaxed for other configurations. The simple case that corresponds to $d>R_{P}+R_{C}$, based on the positions and the transmission powers, the overlapping does not exist (i.e. the intersection is an empty set). Additionally, when one of the disks is totally included in the other, the overlap region equals the surface of the smallest disk. In practice, such configuration means that the active primary receivers presented inside the PR transmitter's disk are interference-free. 


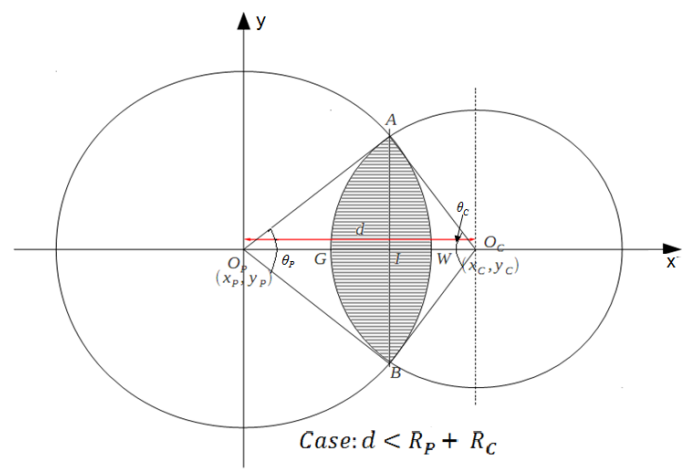

Fig. 1: General overlap case between PR transmitter and a CR node

The general case highlighted in Fig. 1, is obtained when the following condition is satisfied.

$$
d<R_{P}+R_{C} \text { and } d>R_{P} \text { and } d>R_{C}
$$

Using classical geometry, we calculate the general overlap region between $\mathrm{PR}$ and $\mathrm{CR}$ for this case in equation (2).

$$
\begin{aligned}
A_{\text {Overlap }} & =\frac{\theta_{C}}{2} R_{C}^{2}-R_{C}^{2}|\cos \beta| \cos \frac{\theta_{C}}{2} \\
& +\frac{\theta_{P}}{2} R_{P}^{2}-R_{C}|\cos \beta| R_{P} \cos \frac{\theta_{P}}{2}
\end{aligned}
$$

Where $\theta_{C}$ and $\theta_{P}$ are the angles formed at $O_{C}$ and $O_{P}$ with points $A$ and $B$ respectively, whereas $\alpha$ and $\beta$ are intermediate variables for our computation given below

$$
\begin{aligned}
& \sin \alpha=\frac{x_{C}}{\sqrt{x_{C}^{2}+y_{C}^{2}}} \\
& \sin \beta=\frac{R_{P}^{2}-\left(R_{C}^{2}+x_{C}^{2}+y_{C}^{2}\right)}{2 * R_{C} * \sqrt{x_{C}^{2}+y_{C}^{2}}} \\
& \sin \frac{\theta_{C}}{2}=\frac{R_{C}}{R_{P}} *|\cos \beta| \\
& \sin \frac{\theta_{P}}{2}=|\cos \beta|
\end{aligned}
$$

The size of the overlap region depends on the coverage area of both the CR and the PR transmitters on a specific channel. Therefore, determining the size of the overlap takes a considerable part in characterizing the impact on the primary receivers. The smaller the region is, the less impact there is on to the potential PR receivers operating on that channel. As long as the PR receivers are envisaged and protected, the impact that the CRs transmissions have on PR communications can be contained.

\section{Performance EVAluation}

\section{A. Simulation parameters}

The relationship between the overlap phenomena and its impact to the PR network is investigated using OMNeT++ simulator with the integrated MiXim framework. The simulation was deployed in a playground size $3000 \mathrm{~m} \times 3000 \mathrm{~m}$, the PR transmitter a typical $802.11 \mathrm{~b}$ single channel sender with the maximum transmission power of $110.11 \mathrm{~mW}$ that corresponds to a coverage range of $475.8 \mathrm{~m}$. We developed a CR node on top of an adaptative 802.11 multi-channel implementation that allows nodes to switch between channels under specific conditions. Note here that the IEEE 802.11 was selected for simplicity reasons; however, our results can be generalized over any spectrum band. CR transmission power was fixed at $55.11 \mathrm{~mW}$ that covers a distance of $377.77 \mathrm{~m}$. This coverage distance is computed according to the maximum transmission power, wave length and a threshold for the minimal receiving power. The built-in path loss propagation was used with default path loss coefficient alpha of 3.5. All the physical values were also kept by default. The transmission power of the CR node was modified and set to be lower than the PR's transmission power. We placed the PR receivers in the disk area around the PR transmitter following various distributions as we show further. Every shown result is the outcome of 27 simulation runs according to the number of PR receivers and its distribution. Each run consists of at least $500 \mathrm{~s}$. The data were collected and analyzed with $95 \%$ confidence intervals.

\section{B. Simulation scenarios and results}

The experiment characterizes how the PR receivers inside the disk are impacted by the size of the overlap area. We performed the experiment on single channel where the CR and the PR circles overlapped and the PR receivers randomly presented within the PR's disk. Besides, we also tried the topology where the CR's disk overlapped with two other PR's disks which were operating on two different channels as illustrated in Fig. 2

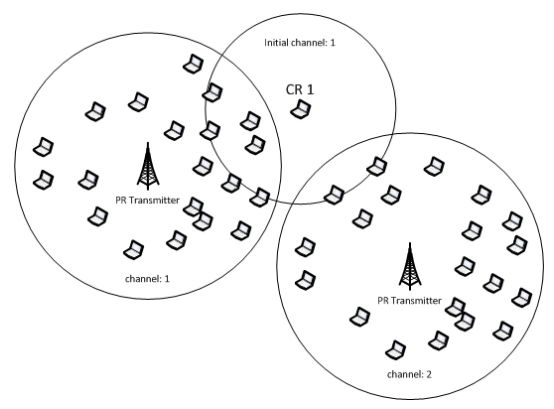

Fig. 2: Multi-channels topology

Fig. 3, Fig. 4 show results for the first scenario where over a single channel network we modify the overlap size and study its impact on PR receivers. We consider Uniform, Poisson and Pareto distributions to deploy PR receivers around the primary radio emitter. As the reason of page limitation, we only showed the result of the experiments with Uniform and Poisson distributions. The $\mathrm{x}$-axis illustrates the size of the overlap decided by the location of the CR sender and calculated based on equation (2); the $\mathrm{y}$-axis shows the ratio of impacted primary nodes. Unsurprisingly, the ratio of impacted $\mathrm{PR}$ receivers by the $\mathrm{CR}$ transmissions grows linearly from $30 \%$ to more than $90 \%$

However, our surprise comes from the observation that the ratio of affected PRs is almost independent of the number of $P R$ receivers as different number of $P R$ receivers are simulated. This important result may seem intuitive with a uniform 
distribution where nodes are deployed inside the coverage disk with an equal probability, but we show here that with unequal probability distribution (i.e. Poisson), this tendency remains somehow similar.

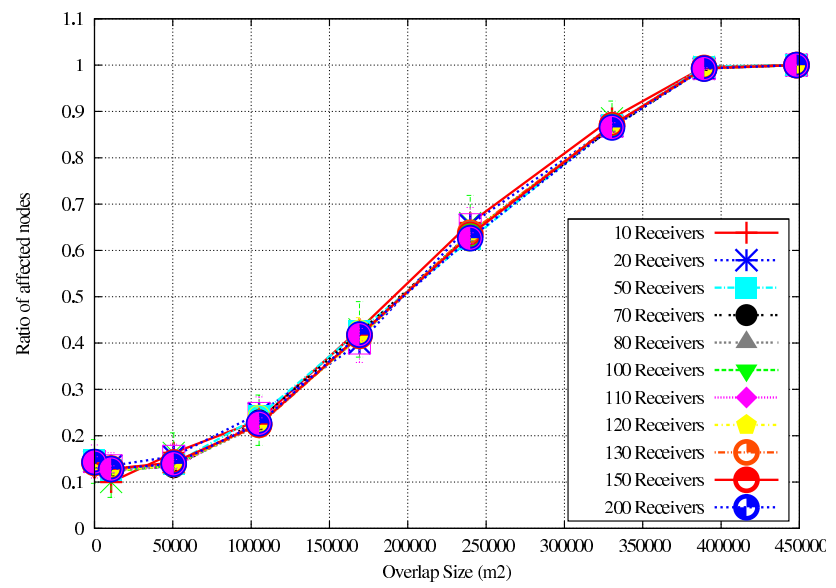

Fig. 3: Single Channel - Different Overlap region - Uniform Distribution

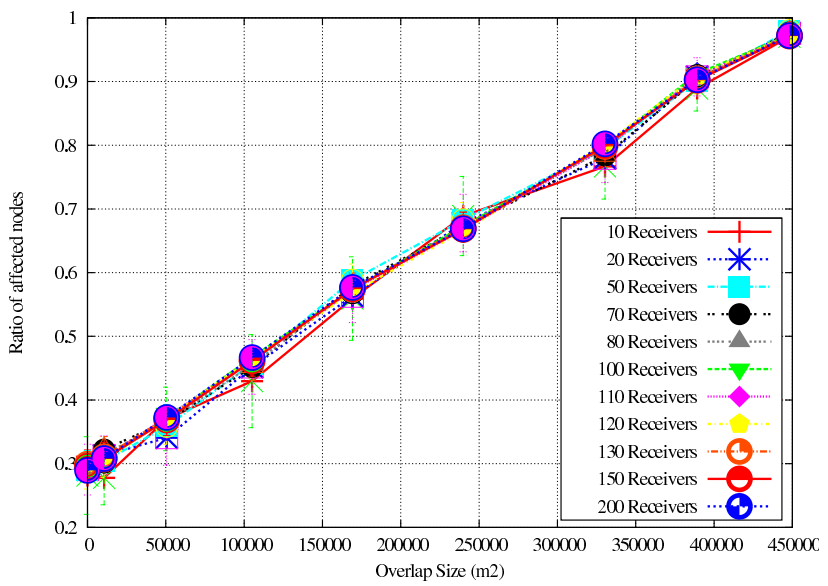

Fig. 4: Single Channel - Different Overlap region - Poisson Distribution

Fig. 5, Fig. 6 correspond to the second scenario, where two channels are available with a CR transmitter observing different overlaps on each channel. In fact, the overlap on channel 1 is around $120000 \mathrm{~m} 2$ while on channel 2 the intersection between transmitter circles is around $500 \mathrm{~m} 2$. The distinctive ratio of affected nodes while modifying the number of PR receivers on both channels. Again, the experiment was performed with various number of PR receivers within the PR transmitter's disks on both channels. The $\mathrm{x}$-axis shows the number PR receivers while the $\mathrm{y}$-axis illustrates the ratio of nodes being affected by the CR transmitter's signal. Obviously, this ratio varies from $10 \%$ to almost $15 \%$ on channel 2 but from almost $34 \%$ to almost $40 \%$ on channel 1 as the receivers are distributed uniformly within these disks (as in Fig. 5). Similar effect for the Poisson distribution is observed in Fig. 6. These results corroborate the previous observation by showing again the limited impact of the number of PRs on the ratio of affected PRs for these distributions (i.e. Uniform and Poisson).

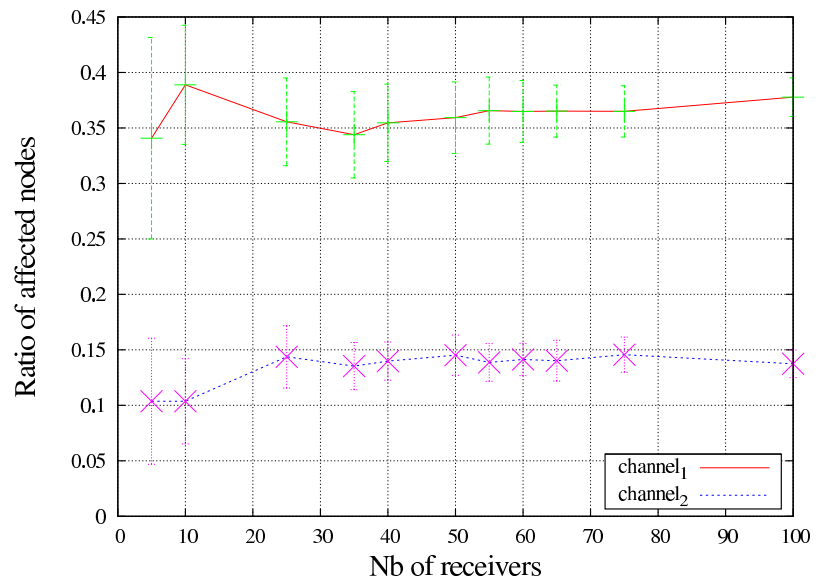

Fig. 5: Multi Channel - Different Overlap region - Uniform Distribution

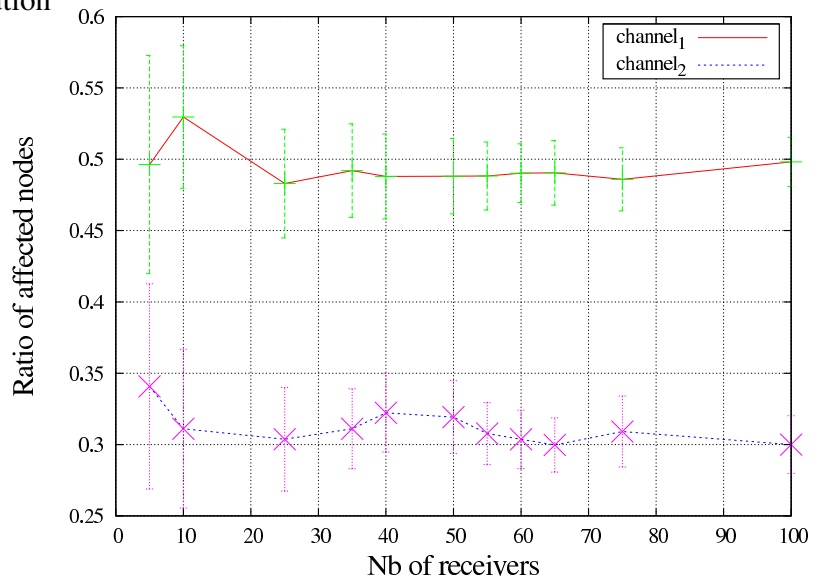

Fig. 6: Multi Channel - Different Overlap region - Poisson Distribution

\section{CONCLUSION AND Future WORK}

In this work, we have studied the impact of secondary users on primary receivers in cognitive radio networks. Based on the position of the primary and cognitive emitters and their transmission power, we have computed the size of the overlap area where PR receivers experience interference. In our future work, we plan to extend our mathematical formulation to include complex cases where multiple PR and CR senders are active as well as other propagation models. Moreover, the applicability of this computation to TV white space will also be considered. Its incorporation in a complete channel selection and routing scheme will also be investigated.

\section{REFERENCES}

[1] H. N. Vu and H. Y. Kong, "Overlap cooperative spectrum sensing with energy comparing in cognitive radio networks," in Strategic Technology (IFOST), 2010 International Forum on, oct. 2010, pp. $230-235$.

[2] E. Bedeer, M. Marey, O. Dobre, and K. Baddour, "On partially overlapping coexistence for dynamic spetrum access in cognitive radio," in 2011 IEEE 16th Workshop on Computer Aided Modeling and Design of Communication Links and Network (CAMAD), 2011.

[3] K. R. Chowdhury and I. F. Akyildiz, "CRP: A Routing Protocol for Cognitive Radio Ad Hoc Networks," IEEE JSAC, (Journal of Selected Areas in Communications), Special Issue on Cognitive Radio Networking and Communications, vol. 29, no. 4, April 2011. 